\title{
Elaboração e desenvolvimento de vídeo educacional em saúde "Conhecendo a
}

\section{gastrostomia"}

\author{
Elaboration and development of educational video in health "Knowing gastrostomy" \\ Elaboración y desarrollo de video educativo en salud "Conociendo la gastrostomía"
}

Marcely Ribeiro Lengruber

ORCID: https://orcid.org/0000-0001-9824-2523

Hospital Adventista Silvestre, Brasil

E-mail: marcely.mestrado@gmail.com

Eliza Cristina Macedo

ORCID: https://orcid.org/0000-0001-8824-9107

Universidade Federal do Estado do Rio de Janeiro, Brasil

E-mail: macedo.unirio@gmail.com

Danielle Galdino de Paula

ORCID: https://orcid.org/0000-0002-0103-6828

Universidade Federal do Estado do Rio de Janeiro, Brasil

E-mail: danigalpa@gmail.com

Mariana Pinheiro Brendim

ORCID: https://orcid.org/0000-0003-2892-462X

Universidade Federal do Estado do Rio de Janeiro, Brasil

E-mail: marianamarinho@edu.unirio.br

Karinne Christinne Cunha

ORCID: https://orcid.org/0000-0003-4971-9801

Universidade Federal Fluminense, Brasil

E-mail: karinne.cunha@hotmail.com

Luiz Eduardo Batista Mendes

ORCID: https://orcid.org/0000-0001-9255-4489

Instituto Militar de Engenharia, Brasil

E-mail: luizeduardobm00@gmail.com

Maria Natalia Pereira Ramos

ORCID: https://orcid.org/0000-0002-8448-1846

Universidade Aberta de Lisboa, Portugal

E-mail: maria.ramos@uab.pt

Mariel Patrício de Oliveira Junior

ORCID: https://orcid.org/0000-0001-9561-2162

Universidade Federal do Estado do Rio de Janeiro, Brasil

E-mail: marieljunior@yahoo.com.br

\begin{abstract}
Resumo
O presente artigo tem como objetivo descrever a criação e o desenvolvimento de um vídeo educativo em saúde elaborado em plataforma digital a fim de contribuir com a educação em saúde sobre a gastrostomia. Considerando que a educação em saúde são saberes destinados ao desenvolvimento humano, o vídeo educativo caracteriza-se como produto de apoio e suporte com fins didáticos na mediação do processo de ensino e aprendizagem em diferentes contextos educacionais. Foram avaliadas seis plataformas, Moravi, Promo-Brazil, Animoto.com, Sparkol, Doodly.com e VideoScrib, Selecionamos a plataforma VideoScribe para execução do projeto. Desejamos que este produto venha somar para a educação em saúde a respeito da temática, contribuindo para a melhoria na qualidade de vida dos pacientes.
\end{abstract}

Palavras-chave: Mídia audiovisual; Gastrostomia; Cuidadores familiares; Ensino.

\begin{abstract}
This article aims to describe the creation and development of a health educational video prepared on a digital platform in order to contribute to health education on gastrostomy. Considering that health education is knowledge intended for human development, the educational video is characterized as a support product with didactic purposes in the mediation of the teaching and learning process in different educational contexts. Six platforms were evaluated, Moravi, Promo-Brazil, Animoto.com, Sparkol, Doodly.com and VideoScrib. We selected the VideoScribe platform to execute the project. We hope that this product will add to health education on the subject, contributing to improving the quality of life of patients.
\end{abstract}

Keywords: Audiovisual media; Gastrostomy; Family caregivers; Teaching. 


\begin{abstract}
Resumen
Este artículo tiene como objetivo describir la creación y desarrollo de un video educativo en salud elaborado en una plataforma digital con el fin de contribuir a la educación sanitaria en gastrostomía. Considerando que la educación para la salud es un conocimiento destinado al desarrollo humano, el video educativo se caracteriza por ser un producto de apoyo con fines didácticos en la mediación del proceso de enseñanza y aprendizaje en diferentes contextos educativos. Se evaluaron seis plataformas, Moravi, Promo-Brasil, Animoto.com, Sparkol, Doodly.com y VideoScrib, seleccionamos la plataforma VideoScribe para ejecutar el proyecto. Esperamos que este producto se sume a la educación sanitaria sobre el tema, contribuyendo a mejorar la calidad de vida de los pacientes.
\end{abstract}

Palabras clave: Medios audiovisuales; Gastrostomía; Cuidadores familiares; Ensenãnza.

\title{
1. Introdução
}

Este material é parte dos produtos oriundos da dissertação de Mestrado profissional realizado na Universidade Federal do Estado do Rio de Janeiro intitulado: "Desenvolvimento e validação de tecnologias educacionais em saúde para o familiar cuidador e o paciente em uso de gastrostomia".

A educação em saúde, por intermédio das tecnologias educativas em saúde, tem como alvo favorecer o processo de cuidado dos indivíduos, se constituindo em uma ferramenta que potencializa a prevenção e proteção de pacientes usuários de dispositivos de alimentação de longa permanência a gastrostomia, podendo ser considerada como processo político pedagógico, permitindo ações transformadoras que levem o indivíduo à sua autonomia e emancipação como sujeito histórico e social, capaz de opinar nas decisões de saúde para cuidar de si, de sua família e de sua coletividade (Frota et al., 2019, p. 1532).

A utilização de recursos audiovisuais, como um vídeo educativo, representa uma evolução na relação ensinoaprendizagem. A utilização das tecnologias em saúde almeja o aperfeiçoamento da prática do cuidado, considerando as atividades técnico assistenciais e burocrático administrativas, bem como as relações interpessoais estabelecidas entre os diferentes indivíduos envolvidos (Moreira et al., 2018, p. 14).

Considerando que a educação em saúde são saberes destinados ao desenvolvimento humano, o vídeo educativo caracteriza-se como produto de apoio e suporte com fins didáticos na mediação do processo de ensino e aprendizagem em diferentes contextos educacionais (Brasil, 2019).

Deste modo, ao utilizar o recurso audiovisual, pode-se oferecer ao público uma base sólida de conhecimentos para que ele compreenda melhor as informações, tenha a capacidade de formar uma opinião crítica sobre determinado tema e anseie transformar o ambiente em que está inserido, visando uma melhor qualidade de vida (Lopes et al., 2018, p. 432).

A partir do exposto, o presente artigo tem como objetivo descrever a criação e o desenvolvimento de um vídeo educativo em saúde elaborado em plataforma digital a fim de contribuir com a educação em saúde para pacientes usuários de gastrostomia e seus familiares cuidadores.

\section{Metodologia}

Trata-se de um estudo metodológico, de natureza qualitativa (Polit \& Beck, 2011; Pereira et al., 2018), do tipo desenvolvimento de tecnologia educativa audiovisual em saúde, elaborado em três fases: pré-produção, produção, pósprodução.

Os aspectos éticos estão sob o regimento da Resolução nº 466/2012 do Conselho Nacional de Saúde (CNS) e foi submetido à apreciação do Comitê de Ética em Pesquisa (CEP) da Universidade Federal do Estado do Rio de Janeiro (UNIRIO). A pesquisa se iniciou após o recebimento do parecer de aprovação nº 4.243.311.

A pré-produção compreendeu o processo de elaboração do storyboard, que consiste em uma série de desenhos ou imagens mostrando a ordem planejada das imagens (Cambridge Dictionary, 2021). 
A construção do roteiro do vídeo foi elaborada a partir de conteúdo com linguagem simples e acessível, previamente validado por juízes profissionais de saúde com expertise sobre o tema, utilizando-se como critério de seleção dos juízes um modelo modificado, proposto por Fehring (Diagnostic Content Valitation, 1994), como segue:

Enfermeiros estomoterapeutas e/ou que tenham pelo menos dois anos de experiência atuando com pacientes gastrostomizados.

Médicos gastroenterologistas ou nutrólogos ou que tenham pelo menos dois anos de experiência atuando com pacientes gastrostomizados.

Nutricionistas especialistas em terapia nutricional ou que tenham pelo menos dois anos de experiência atuando com pacientes gastrostomizados.

Fonoaudiólogos especialistas na área hospitalar ou que tenham pelo menos dois anos de experiência atuando com pacientes gastrostomizados.

Para essa validação foi empregado o método Delphi (Massaroli et al., 2017), uma forma de obtenção de opiniões e critérios de um grupo de especialistas sobre um assunto, utilizando-se da aplicação de questionários, sendo que em cada fase as informações das fases anteriores foram utilizadas em busca do consenso entre os especialistas. Esta técnica permite ainda que o número de especialistas seja determinado diretamente pelo fenômeno que se pretende estudar.

Quanto ao método para calcular a concordância entre os participantes, foi utilizado a porcentagem de concordância absoluta (Alexandre \& Coluci, 2011), que consiste no cálculo do número de vezes que os profissionais concordaram dividido pelo número total de itens a ser avaliado (varia entre 0 e 100\%). O valor de 75\% é considerado o mínimo de concordância aceitável; já valores a partir de $90 \%$ são considerados altos. É considerado consenso, na aplicação de Delphi, quando duas categorias contíguas recebem mais do que dois terços de respostas afirmativas. Neste estudo foi definido o valor de $80 \%$ para concordância aceitável e foram consideradas validadas as respostas marcadas com classificação "1" (Totalmente Adequada) e “2” (Adequada). Questões que receberam classificações 3 (Parcialmente Adequada), 4 (Inadequada) e 5 (Não se aplica), retornaram em segunda rodada aos profissionais, conforme as respectivas sugestões e com as alterações pertinentes, para nova avaliação e obtenção do maior consenso de adequação entre os profissionais.

A seleção dos especialistas foi feita por amostragem não probabilística do tipo conveniência, isto é, o pesquisador selecionou os elementos de uma amostra a que tem acesso, admitindo que esses possam representar um universo (Oliveira, 2001). Isso foi operacionalizado por meio dos nomes e contatos de profissionais da área com potencial perfil para participarem do estudo, indicados pelos docentes e participantes dos grupos de pesquisa do Programa de Pós-Graduação em Saúde e Tecnologia no Espaço Hospitalar (PPGSTEH) da Universidade Federal do Estado do Rio de Janeiro (UNIRIO) e a posterior leitura dos respectivos currículos Lattes.

Foi enviada uma carta convite com os objetivos e procedimentos de coleta de dados da pesquisa para os endereços eletrônicos dos profissionais que se adequaram aos critérios estabelecidos. A cada profissional selecionado foi solicitada a indicação de outros participantes.

Quanto ao questionário, foram elaboradas perguntas fechadas, do tipo Likert com cinco alternativas de respostas e uma resposta para cada pergunta, assim representada:

1: totalmente adequado (TA)

2: adequada (A)

3: parcialmente adequada (PA)

4: inadequada (I)

5: não se aplica (NA).

Tanto a carta convite quanto o Termo de Consentimento Livre e Esclarecido (TCLE) e o questionário, foram 
encaminhados por meio da geração de um Link pela ferramenta Google Forms via e-mail, que é uma ferramenta versátil, gratuita e de acesso fácil. O prazo para o retorno das respostas dos profissionais foi estipulado em 10 dias. Para todos os itens foram oportunizados opiniões e apontamentos dos profissionais convidados a participar. As avalições do conteúdo pelos profissionais juízes ocorreram nos meses de setembro e outubro de 2020.

Na primeira rodada do método Delphi o conteúdo para avaliação foi enviado para 81 participantes, destes, 41 responderam. A partir daí, foi realizado uma devolutiva para todos os participantes, conforme exigido pelo método Delphi, por meio de e-mail, sobre as observações feitas. Realizaram-se os ajustes pertinentes e estes foram reenviados aos respectivos profissionais juízes.

Os dados gerados nas duas rodadas foram analisados pelo Índice de Validade de Conteúdo (IVC).

Fórmula 1. Índice de Validade de Conteúdo.

\begin{tabular}{|c|c|c|}
\hline & $\begin{array}{c}\text { número de } \\
\text { participantes que }\end{array}$ & SOMA (IVCs) \\
\hline \% concordância = & $\frac{\text { concordaram }}{\text { número total de }} \times 100$ & Número de itens avaliados \\
\hline
\end{tabular}

Fonte: (Alexandre \& Coluci, 2011).

\section{Resultados e Impacto do Produto}

Como resultado obteve-se para a $1^{\circ}$ rodada IVCG de $93 \%$ e na segunda rodada de avaliação do conteúdo IVCG de 97\%. Como aceitável considerou-se $80 \%$ tanto para a avaliação de cada item como para a avaliação geral do conteúdo.

Quanto a busca e seleção de plataformas digitais para produção de material audiovisual foram visitadas as seguintes: Moravi, Promo-Brazil, Animoto.com, Sparkol, Doodly.com e VideoScrib, optando-se pela última. Todas essas plataformas apresentam a possibilidade de uso gratuito, por meio do download de uma versão de teste com número específico de dias para a utilização, em geral para uso por sete dias, porém com limitações de acesso a determinados recursos. A assinatura das plataformas digitais, no entanto, permite o acesso mais amplo aos recursos disponíveis como, por exemplo: banco de imagens e arquivos de áudios.

A produção compreendeu aspectos de seleção de figuras e imagens do banco de imagens da plataforma e originais da autora, disponibilizando ainda a seleção de música, tipo e cor de letra, fundo, gravação do áudio (foi gravado em voz feminina e masculina) e ajuste das imagens, do tempo, da sequência de surgimento na tela como o curso de movimento do vídeo, esses últimos se constituíram, no processo, a parte mais trabalhosa da produção.

Na pós-produção o vídeo foi finalizado com duração de dois minutos, e dezessete segundos em tela contínua com 28 imagens e figuras, áudio gravado com vozes masculina e feminina em ambiente doméstico e trilha sonora da plataforma.

É recomendado que este tipo de ferramenta educacional não ultrapasse 15 minutos, já que após este período a manutenção da atenção de quem o assiste torna-se comprometida. Nesse sentido, entende-se que a versão final do vídeo elaborado neste estudo condiz com que é recomendado na literatura (Fleming et al., 2009).

"A elaboração de vídeo educativo pode possibilitar ao profissional de saúde formas inovadoras de educação pois aproxima os conteúdos abordados da realidade, além de despertar interesse e promover melhor aprendizado" (Andrade \& Fernandes, 2017, p. 765).

Considera-se que essa tecnologia poderá ser utilizada em conjunto com outras ferramentas educativas desmistificando assim as dificuldades e dúvidas a respeito da GTT e potencializando práticas colaborativas de aprendizagem autônoma, sendo 
estas apresentadas por meio de tecnologias de informação e comunicação (Coelho Neto et al., 2019).

$\mathrm{O}$ uso de vídeos educativos pode ser uma estratégia que proporciona ao indivíduo e à sua família cuidadora, a construção de saberes relacionados à doença enfrentada ou ao seu tratamento, aqui, neste caso, a gastrostomia, pois o vídeo é uma tecnologia que pode ser aplicada à resolução de um problema (Stragliotto et al., 2017).

Cada recurso tecnológico tem uma finalidade distinta a ser alcançada. Nesse contexto, os vídeos educativos, poderão contribuir com as políticas públicas brasileiras no âmbito da promoção da saúde, disseminando o conhecimento de maneira didática. Este produto visa impactar positivamente tanto no ambiente hospitalar como no domiciliar, por meio da facilitação no processo de aceitação do dispositivo da gastrostomia e no preparo do paciente e do cuidador para os cuidados com o dispositivo na rotina domiciliar.

\section{Considerações Finais}

Esse vídeo educativo pretende preencher a lacuna do conhecimento entre pessoas leigas como um instrumento didático e tecnológico para a promoção da saúde, já que reúne elementos de fácil compreensão por meio de uma dinâmica criativa.

A validação de um conteúdo pela equipe multiprofissional de saúde para a realização de algo inovador para a sociedade é algo que contribui para o conhecimento e desenvolvimento das ações interdisciplinares, além de facilitar e potencializar o processo de educação, conhecimento e transformação social, contribuindo para a melhoria na qualidade de vida de pacientes usuários de gastrostomia e seus familiares cuidadores. Além do mais, que possa somar ideias e informações para outros projetos de promoção da saúde e prevenção de danos.

Deseja-se que este produto venha somar para a educação em saúde a respeito da temática, contribuindo para a melhoria na qualidade de vida dos pacientes.

\section{Agradecimentos}

Contribuíram com a produção deste vídeo Virgílio Gomes do Nascimento e Guilherme Vinícius Guimarães do Nascimento.

\section{Referências}

Alexandre, N. M. C., \& Coluci, M. Z. O. (2011). Validade de conteúdo nos processos de construção e adaptação de instrumentos de medidas. Ciência \& Saúde Coletiva, 16(7), 3061-3068. 10.1590/S1413-81232011000800006

Andrade, M. E., \& Fernandes, M. C. (2017). Vídeos interativos como ferramenta de socialização de saberes: vivências no uso de tecnologias educacionais no processo formativo. Revista de Pesquisa Interdisciplinar, 2(Supl.), 765-769. 10.24219/rpi.v2i2.0.314

Considerações sobre Classificação de Produção Técnica e Tecnologia (PTT): Enfermagem. Brasília: Ministério da Educação. http://www1.capes.gov.br/images/FICHA_AVALIACAO/ENFERMAGEM_CRITERIOS_PRODUCAO_TECNICA.pdf

Cambridge Dictionary. (2021). Storyboard. Cambridge: Cambridge $\quad$ University https://dictionary.cambridge.org/pt/dicionario/ingles/storyboard?q=STORYBOARD

Coelho Neto, J., Marcomini, J. T. S., \& Bueno, L. G. (2019). O uso das tecnologias digitais no contexto de aprendizagem: scratch, logo e objetos de aprendizagem. Research, Society and Development, 8(12), e078122574. 10.33448/rsd-v8i12.1574

Fleming, S. E., Reynolds, J., \& Wallace, B. (2009). Lights camera action! a guide for creating a DVD/video. Nurse Educator, 34(3), 118-121. 10.1097/NNE.0b013e3181a0270e

Frota, K., Santos, L. T. S., Oliveira, L. S., Marques, M. F., \& Ponte, K. M. A. (2018). Tecnologias educativas: estratégias eficientes para a promoção da saúde de idosos. Revista Saúde.com, 15(2), 1531-1537. 10.22481/rsc.v15i2.4401

Lopes, V. C. A., Moura, E. C. C., Sales, I. M. M., \& Rocha, S. S. (2018). Educational video for promoting men's health: a descriptive comparative study. Online Brazilian Journal of Nursing, 16(4), 431-438. 10.17665/1676-4285.20175854 
Research, Society and Development, v. 10, n. 3, e23210313060, 2021

(CC BY 4.0) | ISSN 2525-3409 | DOI: http://dx.doi.org/10.33448/rsd-v10i3.13060

Massaroli, A., Martini, J. G., Lino, M. M., Spenassato, D., \& Massaroli, R. (2017). Método Delphi como referencial metodológico para a pesquisa em enfermagem. Texto \& Contexto-Enfermagem, 26(4), e1110017. 10.1590/0104-07072017001110017

Moreira, T. M. M., Pinheiro, J. A. M., Florêncio, R. S., \& Cestari, V. R. F. (2018). Tecnologias para a promoção e o cuidado em saúde: EdUECE.

Oliveira, T. M. V. (2001). Amostragem não probabilística: adequação de situações para uso e limitações de amostras por conveniência, julgamento e quotas. Administração Online - prática - pesquisa - ensino, 2(3), 1-15. https://pesquisa-eaesp.fgv.br/conveniencia.pdf

Pereira, A. S., Shitsuka, D. M., Pereira, F. J., \& Shitsuka, R. (2018). Metodologia da pesquisa científica. UFSM. https://repositorio.ufsm.br/bitstream/handle

Polit, D. F., \& Beck, C. T. (2011). Fundamentos de pesquisa em enfermagem: avaliação de evidências para as práticas de enfermagem: Artmed.

Stragliotto, D. O., Girardon-Perlini, N. M. O., Rosa, B. V. C., Dalmolin, A., Nietsche, E. A., Somavilla, I. M., \& Silva, M. E. N. (2017). Implementação e avaliação de um vídeo educativo para famílias e pessoas com colostomia. Revista ESTIMA, 15(4), 191-199. 10.5327/Z1806-3144201700040002 